\title{
Computer application in bio-process engineering in Japan
}

\author{
Toshiomi Yoshida \\ International Center for Biotechnology \\ Osaka University \\ Osaka, Japan
}

\section{Intelligent control of bio-processes}

$\mathrm{D}$ uring the last 50 years of the modern fermentation technology development, bio-process engineering has made rapid progress along with the remarkable development of computer science and technology in the fields of both hardware and software. In earlier days modeling and simulations were carried out for the optimization of bio-processes utilizing the traditional approaches of system analyses, including the expression of biological phenomena using deterministic mathematical models at the levels of culture, cell, metabolic network, enzymatic reactions and gene expression. With the aid of powerful theoretical and mathematical methods of optimization such as Greens theorem, Pontryagins maximum principle, Bellmans dynamic programming, numerous attempts of process optimization were made to provide us with many interesting results of analyses and useful suggestions for process operations. During the last 10 years, extensive research has been carried out on the intelligent control of bio-processes. There has been a trend towards interpretation of the problems involved in the control of bio-reactor systems from informal viewpoints, using nontraditional methods, artificial intelligence techniques or knowledge engineering approaches. This paper deals with the recent developments in the area of computer applications in bio-process engineering in Japan, specially focusing on knowledge engineering approaches.

\subsection{Optimization of bio-processes}

Optimization of cultivation processes has been attempted in two ways, one based on deterministic models of differential equations or algebraic equations, and the other approaches without the use of mathematical equations. Optimization based on mathematical models has been investigated to demonstrate typical examples of the application of the optimization theories. Despite many studies on the utilization of these methods, including minimi- 
zation of settling time, maximization of cell production, and maximization of product formation, we have seen few examples of direct application of the results of such works at production sites. Two major reasons are pointed out for the difficulties of utilizing these methods in practice. One is the gap between mathematical models and real processes, and the other is the way of evaluation - a single objective function for very sophisticated real problems. To overcome the problem of the gap we have tried various approaches without the use of deterministic mathematical models. A possible method for resolving the problems might be the application of knowledge-based approaches. The optimization of fermentation processes aimed at quality control including the sensory analysis is a typical example of challenging problems with difficulty of modeling. Recent investigations of fermentation control of sake mashing using the fuzzy theory (1-8) might be a good example encouraging us to use knowledge-based approaches.

\subsection{Monitoring and diagnosis}

For realization of optimization or even quasi-optimization such as the set-point control of RQ, it is necessary to devise a measurement and control system. A measurement system monitors the change of state variables, either directly or indirectly. The direct method uses sensors to measure observable variables and indirect methods use software sensors for estimation of nonobservable variables based on various types of information involving measurement of observable variables and even biological and biochemical knowledge. The number of accurate, reliable, but inexpensive sensors, especially for industrial microbial cultivation processes, on natural complex media is rather limited. It is inevitable that the use of software sensors requires information on cellular physiology and metabolism.

\subsection{Process control}

Bio-processes are usually operated in batch or fed-batch modes, and continuous culture methods are rarely applied. Thus, the state of process changes dynamically in the course of cultivation. However, it is not possible in many cases to directly measure control objectives such as product concentration or production rate. Bio-processes possess various characteristics which perplex us in establishing control systems. Mathematical models are non-linear with time-variable parameters; a limited number of operational variables are available in spite of many control objectives; quick and precise control is required in spite of large time constants of the process dynamics; the variations of strain characteristics and cultivation behavior (perturbation of initial conditions) are rather extensive. Consequently sophisticated control systems are required in order that the processes and application of knowledge engineering approaches should become popular.

To fill the gap between mathematical models and real phenomena, the combined application of knowledge engineering approaches such as the fuzzy 
inference techniques, expert systems, and artificial neural networks, has been attempted in the process control of microbial cultivation. Since bioreactor systems inherently include complex biological networks, they are regarded as multi-variable and multi-structure systems from the viewpoint of process control. There are many events which can be qualified as structurealtering phenomena, such as changes of metabolic pathways, diauxic growth. expression or repression of genes caused by chemical factors or temperature. and spore formation. Since these physiological phenomena result in alter ations of the internal structures of the systems, consequent alterations the control system structure are required. Consequently, a multi-structu control system with a hierarchical structure is proposed for application the control of bioreactor systems. The control strategy is capable of dynamic alterations according to the current process situation, generally the physiological situation of the cell population (9). Thus, the fuzzy inference technique has been applied for the recognition of cultivation phases (10-13).

\section{Fuzzy inference and control}

In the field of bio-process engineering Filev et al. (14) first applied the fuzzy inference technique for the simulation of a fermentation process. Thereafter, the fuzzy inference technique has proved a powerful tool for identification of the physiological state, or culture phase during cultivation of microbial cells, and remarkable progress in the development of methods for on-line monitoring and automatic control of bio-processes has been made using the fuzzy theory, and their effectiveness has been proved by their applications to commercial processes.

\subsection{Simulation of processes}

A linguistic model was proposed by Filev et al. (14) for simulation of a batch fermentation process, namely, alcohol production by yeast. The fuzzy theory was applied based on using qualitative characteristics in order to reduce the uncertainty of quantitative description of the processes. A combination of fuzzy inference for culture state diagnosis and a statistical approach for parameter estimation has been claimed as a satisfactory method for the simulation of microbial cultivation.

\subsection{Identification of physiological situation or culture phase}

Identification of the culture phases in batch operations, such as lag phase, growth phase, production phase, and declining phase including sporulation phase or other inactivating cell activities toward the end of the batch culture, can be made using the fuzzy inference procedure (10). Horiuchi, et al. (12) developed a control system based on phase identification by fuzzy inference. Further studies (15) were carried out on an integrated 
system for phase diagnosis by fuzzy inference, and optimized control depending on the culture phase identified. Shimizu, et al. (16) developed a method for culture state diagnosis and parameter estimation utilizing the fuzzy inference procedure in combination with molar-flux calculations based on a metabolic reaction model. Diagnosis of culture state by fuzzy inference was made after investigation of the yeast growth and ethanol production leading to precise determination of the state of high ethanol production in spite of a low growth rate. For the estimation of growth rate under conditions of metabolic imbalance which hinder accurate estimation of molar fluxes and growth rate, an approach allowing for variations in culture state was developed using the idea of altering the oxidative phosphorylation parameter, $\mathrm{P} / \mathrm{O}$.

\subsection{On-line monitoring of state variables}

During cultivation, the cell population often undergoes physiological transformations which can be interpreted as structural alterations of the process from the process system engineering aspect. For the diagnosis of such changes of process structure, it is required to select and calculate a set of variables, which provide an adequate basis for the detection of structural alterations using the pattern recognition theory. As a software sensor, fuzzy inference has been reported to be powerful for monitoring the physiological state $(10,17-20)$.

\subsection{Process diagnosis and control}

Structural variability is a fundamental property of biological systems. To overcome the problem of conventional control systems which are based on a single-structure plant, we offered an expert approach, a two-level hierarchical scheme, for the control of bio-processes (10). At the higher level, which performs the organizing functions and operates in the structural space of the plant, the current plant structure is recognized as an element of a finite set of structures defined on the basis of expert knowledge of the characteristics of the microbial population. The recognition is based on fuzzy decomposition of the structural space of the plant into several subspaces, mapped isomorphically into a respective set of rational control strategies. Two distinct functions were assigned to the lower level. One performs the global task of control in its structural space. The other function has a local character and consists of control of the plant in a particular subspace of constant structure. The proposed approach has been realized as a real-time software system, with enough flexibility to be used with different fermentation processes.

One of the most typical examples of the effective use of fuzzy theory on process control is fuzzy control of sake mashing processes (1-4,8). Recently Kobayashis group (5-7) has extensively studied fuzzy control of mashing process of a sake, of particularly high quality and traditionally appreciated, 
called, ginjo-shu. The fuzzy rules for process control of ginjo-shu mashing were developed based on an expert of sake mashing, tohji. Fuzzy control has been successfully applied in many production plants. Control of the dissolved oxygen (DO) level was performed in a fuzzy control scheme with manipulation of aeration and agitation rate taking into account the time increment of DO (21). Feeding of substrate has been extensively investigated as a target of fuzzy control in commercial plants such as the production of the precursor of pravastatine (22). Okada et al. (23) reported that a low productivity syndrome of an antibiotic, milbemycin, caused mainly by insufficient oxygen supply in a commercial-scale fermentor, could be overcome by the consistent and reliable actions of fuzzy control system. Shioyas group (24) presented a method of applying fuzzy logic on a compensator in a scheme of feed-forward/feedback controller to realize utilization of non-linearity for robustness of control, flexibility in use of multiple measurable variables, and easy and effective use of expert knowledge.

\section{Construction and application of expert systems}

Expert systems have been applied to the control of bio-processes to provide a means of imitating thoughts and decisions by a skilled engineer with long years of experience in the operation of bio-processes. Sophisticated expert systems have been realized owing to significant developments in computer technologies. Modern expert systems have been constructed using a tool called expert system shell. The shell consists of an editor for incorporating and editing various knowledge, a knowledge-base for logging the processed knowledge, an inference engine for various inferences utilizing the knowledge-base, and a user interface to facilitate the communication with a non-expert user. The shells developed for process control should have an interface installed to communicate with the control sections.

Several examples of expert systems have been developed in Japan. Konstantinov et al. $(10,17)$ first demonstrated an example of utilization of the concept of the expert system for diagnosis of the process structure of bioprocesses possessing physiological state variability, using knowledge-based approaches to express the diversity of the process structure of bio-processes. Another example of the use of the expert system approach was presented by Kishimoto, et al. (25), who studied the optimization of feeding of penicillin in the production of glutamic acid by constructing an expert system and using a fuzzy relationships scheme to show the utility of fuzzy theory in automatic determination of the policies in operation of bio-processes. An expert system for image analysis was also developed by Fukuda et al. $(26,27)$ to show a satisfactory performance when applied to the detection and sizing of animal cells grown on micro-carriers. Siimes et al. (28) demonstrated the success of an object-oriented fuzzy expert system for on-line diagnosis and control of bio-processes. Nakajima et al. (29) developed a general-purpose expert shell for diagnosis and control of bio-processes, and showed the utility 
of the system for on-line diagnosis and control, applying it to lactic acid fermentation for detection of seed inferiority.

\section{Artificial neural networks for simulation and control}

Artificial neural network, ANN, a black-box type model for estimation and prediction of a set of inputs, is principally composed of units equivalent to neurons and links analogous to synapses, and the input and output signals are correlated using a sigmoid function, or a logistic function. The learning procedure of an ANN, namely tuning of a weight vector for inputs is usually performed using the back propagation method applying a steepest descent method.

Many applications of ANN for the estimation of state change and controls of bio-processes are reported. After the learning procedure estimation of the future state, $y(t+1)$, for a given operational input $u(t)$, is performed using past input-output data, $u(\mathrm{t}-1), \ldots, u(\mathrm{t}-\mathrm{m}), y(\mathrm{t}), y(\mathrm{t}-1), \ldots, y(\mathrm{t}-\mathrm{n})$, and then a control procedure can be made searching $u(t)$ to adjust $y(t+1)$ at a prerequisite value. Karim et al. (30) presented the results of application of an ANN to the control of feeding glutamine and glucose for monoclonal antibody production by a hybridoma cell culture. ANNs of various structures were used depending on the culture phase or physiological situation of cells, and a recurrent type of ANN was experimentally proved to be superior for accurate prediction. Several examples of application of ANN have been found in studies on sake brewing, such as the prediction of culture characteristics (31), cell growth (32), process control (33), and sensory evaluation (34-36). Shimizu's group developed a neuro-fuzzy control scheme combining the advantages of both ANN and fuzzy control and confirmed the utility of the method by applying it to baker's yeast cultivation (37), and recombinant cultures (38). Kobayashi's group (36) applied a fuzzy neural network (FNN) scheme to the sensory evaluation of sake to reduce the time and labor for tuning the membership function of fuzzy inference. The membership function of the premise was tuned by modifying weight function by back propagation, thereby establishing fuzzy rules.

\section{Genetic algorithm}

For the design, operation and control of bio-process systems, fuzzy neural networks, expert systems and their combination have been effectively utilized. Moreover, other intelligent computational methods such as genetic algorithm, wavelet analysis, chaos analysis can also be utilized. Genetic algorithm (GA) has been utilized as a method for searching the optima of combination problems analogous to the mechanism of hereditary transmission and evolution involving: 1) logging of information in genes, 2) succession of information by copying genes, 3) generation of new gene types and expression 
types by mutation, and 4) survival and evolution by competition depending on adaptability to environment.

\subsection{Process optimization}

GA is a method used as an algorithm of combination optimization. For the optimization of time profile of temperature control in a fermentation process, the problem can be reduced to the choice of optimum series of temperatures at segmented culture times (39). In the learning process of development of fuzzy rule or membership function, GA has been often used $(8,40)$. On-line optimization in searching feeding rate with a limited range of nutrients for growth and antibody production by hybridoma was made by GA (41).

\subsection{Quality control}

Ishii et al. (42) reported application of GA on quality control of baker's yeast. Feeding rate of sugar was optimized using a GA adopting an objective function of multi-variables to minimize the deviation of fermentability and cell mass from the target values, and the penalty index of the feed deviation from the maximum feed rate at which fermentative metabolism started. After 250 cycles of searching including reproduction, close-over, and mutation, excellent strains were obtained.

\subsection{Search of fuzzy rules and optimization}

Optimal control of sensory evaluation estimated from the concentrations of 13 components was attempted for mashing of Ginjo-shu (sake) (8). The control system consisted of fuzzy simulators generated by a GA and an optimization procedure based on another GA. The fuzzy simulators simulated the dynamics of the ethanol production rate and sensory evaluation. Decentralized learning of fuzzy rules was also introduced. The fermentation period was divided into 4 phases, with a set of fuzzy rules corresponding to each phase. In order to construct an adaptive system based on the fuzzy simulators, only the set of rules corresponding to the current phase was identified, with the result that the fuzzy rules were adapted to fluctuations in the relationship between the temperature and ethanol production rate. By optimizing control in this way, optimal quality of sake was successfully obtained.

\section{Conclusion}

Various methods of knowledge engineering have been applied for the development of the bio-process control by effective use of computers. After 10 years of experience in this field we are ready to construct a general-pur- 
pose intelligent control system for bio-processes integrating all the technologies we have developed.

\section{Literature}

1. Tsuchiya Y., Koizumi J., Suenari K., Teshima Y., Nagai S., (1990), Constructions of fuzzy rules and a fuzzy simulator based on the control techniques of Hiroshia tohji (experts), Hakkoukogaku Kaishi (Japanese), 68, 123-129.

2. Suenari K., Tsuchiya Y., Teshima Y., Koizumi J., Nagai S., (1990), Performance of sake mash brewing with fuzzy control, Hakkoukogaku Kaishi (Japanese), 68, 131-136.

3. Oishi K., Tominaga M., Kawato A., Imayasu S., Nanba S., (1991), J. Ferment. Bioeng., 72, $115-121$.

4. Imayasu S., Suginami K., Abe Y., Kawado A., Oishi K., (1993), Development of a new sake brewing method using liquefied rice, Seibutu-kogaku Kaishi (Japanese), 71, 29-42.

5. Hanai T., Hondan H., Takahasi N., Nishida T., Fukaya I., Kobayasi T., (1994), Framework rules for control of the sake (Ginjo) making process and their application in fuzzy control, Seibutu-kogaku Kaishi (Japanese), 72, 275-282.

6. Hanai T., Nishida T., Ohkusu E., Honda H., Kobayasi T., (1995), Experimental fermentation of Ginjo sake with two fuzzy controls, Seibutu-kogaku Kaishi (Japanese), 73, 283-364.

7. Nishida T., Fukaya I., Takahasi N., Hanai T., Honda H., Kobayasi T., (1994), Construction of fuzzy rules based on statistically analyzed data for control of the sake (Ginjo) making process, Seibutsu-kogaku Kaishi (Japanese), 72, 267-274.

8. Matsu-ura K., Shiba H., Hirotsune M., Hamachi M., (1995), J. Ferment. Bioeng., 80, 251-258.

9. Konstantinov K., Yoshdia T., (1989), Biotechnol. Bioeng., 33, 1145-1156.

10. Konstantinov K. B., Yoshida T., (1990a), J. Ferment. Bioeng., 70, 48-57.

11. Kishimoto M., Moo-Young M., Allsop P., (1991), Bioproc. Eng., 6, 163-172.

12. Horiuchi J., Kamasawa M., Miyakawa H., Kishimoto M., Momose H., (1993), J. Ferment. Technol., 76, 382-387.

13. Horiuchi J., Kishimoto M., Momose H., (1995), J. Ferment. Technol., 79, 297-299.

14. Filev D. P., Kishimoto M., Sengupta S., Yoshida T., Hisaharu Taguchi, (1985), J. Ferment. Technol., 63, 545-553.

15. von Numers C., Nakajima M., Asama H.,, Linko P., Endo I., (1994), J. Biotechnol., 34, 109-118.

16. Shimizu H., Miura K., Shioya S., Suga K., (1995), Biotechnol. Bioeng., 41, 165-173.

17. Konstantinov K. B., Yoshida T., (1990b), J. Ferment. Bioeng., 70, 420-426.

18. Konstantinov K. B., Nishio N., Seki T., Yoshida T., (1991a), J. Ferment. Bioeng., 71 , 350-355.

19. Konstantinov K. B., Yoshida T., (1991b), A knowledge-based pattern recognition approach for real-time diagnosis and control of fermentation processes as variable structure plants, IEEE Trans. Syst. Man Cybern., 21, 908-914.

20. Konstantinov K. B., Yoshida T., (1992), J. Biotechnol., 24, 33-51.

21. Nakamura T., Kuratani T., Morita Y., (1985), Fuzzy control: Application to glutamic acid fermentation, Proc. of IFAC Modeling and control of Biotechnology Process, 211-215.

22. Hosobuchi M., Fukui F., Matsukawa H., Suzuki T., Yoshidakawa H., (1993), J. Ferment. Technol., 76, 482-486.

23. Okada S., (1995), Effect of fuzzy control on milbemycin production, Seibutsu-kogaku Kaishi (Japanese), 74, 203-205.

24. Alfafara C., Miura K., Shimizu H., Shioya S., Suga K., Suzuki K., (1993), Biotechnol. Bioeng., 41, 493-501.

25. Kishimoto M., Kitta Y., Takeuchi S., Nakajima M., Yoshida T., (1991), J. Ferment. Bioeng., 72, 110-114. 
26. Fukuda T., Shiotani S., Arai F., Asama H., Nagamune T., Endo I., (1991a), Recognition and counting method of animal cells on microcarrier using image processing based on expert system - Improvement of the recognition with the fuzzy inference and the speed-up of the fast image processing with bead-recognition by bead-contour-tracking. Nihon-Kikai-Gakkai- Ronbunshu (C) (Japanese), 57, 826-832.

27. Fukuda T., Shiotani S., Arai F., Asama H., Nagamune T., Endo I., (1991b), Recognition and counting method of biological cells on microcarrier using image processing based on expert system - Recognition of overlapping nonuniform cells by neural network, NihonKikai-Gakkai- Ronbunshu (C) (Japanese), 57, 3847-3854.

28. Siimes T., Nakajima M., Yada H., Asama H., Nagamune T., Linko P., Endo I., (1992), Appl. Microbiol. Biotech., 37, 756-761.

29. Nakajima M., von Numers C., Yada H., Siimes T., Pokkinen M., Endo I., Linko P., (1994), Appl. Microbiol. Biotech., 42, 204-209.

30. Karim M. N., Yoshida T., Rivera S. L., Saucedo V. M., Eikens B., Oh G. S., (1997), J. Ferment. Technol., 83, 1-11.

31. Oishi K., Tominaga M., Kawato A., Imayasu S., (1992), J. Ferment. Bioeng., 73, 153158.

32. Matsu-ura K., Hirotsune M., Hamachi M., (1991b), Cell density identification in alcohol fermentation by neural network, Hakkou-kogaku Kaishi (Japanese), 69, 463-469.

33. Matsu-ura K., Hirotsune M., Nakada F., Hamachi M., (1991a), On-line control for continuous sake fermentation, Hakkou-kogaku Kaishi (Japanese), 69, 455-461.

34. Matsu-ura K., Shiba T., Horitsune M., Nunokawa Y., (1993b), Optimal control on the sensory evaluation of sake mashing process, Proceedings of the PSE 94, Korea, 2, 1081-1089.

35. Kagami A., Hanai T., Honda H., Kobayashi T., Nishida T., Fukaya I., (1995), Estimation of total evaluation from sensory evaluation of sake (Ginjo) using a neural network, Seibutu-kogaku Kaishi (Japanese), 73, 199-205.

36. Kagami A., Hanai T., Honda H., Kobayashi T., (1995), Quality modeling of Ginjo sake using a neural network and genetic algorithm, Seibutu-kogaku Kaishi (Japanese), 73, 387-395.

37. Shi Z., Shimizu K., (1992), J. Ferment. Bioeng., 74, 39-45.

38. Ye K., Jin S., Shimizu K., (1994), J. Ferment. Bioeng., 77, 663-673.

39. Matsu-ura K., Shiba T., Nunokawa Y., Shimizu H., Shioya S., Suga K., (1993a), Calculation of optimal trajectories for fermentation processes by genetic algorithm, Seibutukogaku Kaishi (Japanese), 71, 171-178

40. Nakanishi S., (1994), Fuzzy control by genetic algorithm, System, Control, Information (Japanese), 38, 613-618.

41. Oh G., Takagi M., Yoshida T., (1996), Optimization of hybridoma culture by a combination of neural network and genetic algorithm, Seibutsu-kogaku Kaishi (Japanese), 74, 192194.

42. Ishii N., Shinomiya Y., Ikeshiro A., Ando M., (1996), Quality control of bakers yeast by genetic algorithm, Seibutu-kogaku Kaishi (Japanese), 74, 205-208.

\section{Computer application in bio-process engineering in Japan}

\section{Summary}

Recent development of research conducted in Japan into computer applications in bio-process engineering is summarized, specially focusing on the application of knowledge engineering approaches to bio-process control. The issues of methodological investigation, their applications in process development, and examples of industrial applications of expert systems, artificial 
neural networks, fuzzy theory, and genetic algorithm are thoroughly reviewed, and some representative recent works are introduced more extensively.

\section{Key words:}

intelligent control, fuzzy control, knowledge engineering, neural networks, expert system, genetic algorithms.

Address for correspondence:

Toshiomi Yoshida, International Center for Biotechnology, Osaka University 2-1, Yamada-oka, Suita, Osaka, Japan. 